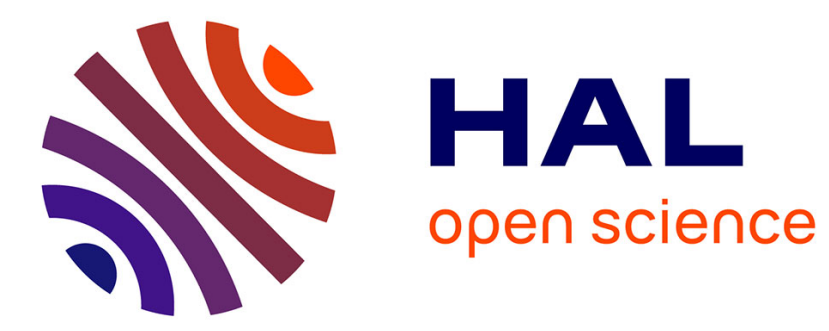

\title{
Cluniacensia bibliographica minima
}

Sébastien Barret

\section{To cite this version:}

Sébastien Barret. Cluniacensia bibliographica minima. Revue Mabillon, revue internationale d'histoire et de littérature religieuses, 2011, n.s. 22 (83), pp.291 - 303. 10.1484/J.RM.5.100998 . halshs01700852

\section{HAL Id: halshs-01700852 \\ https://shs.hal.science/halshs-01700852}

Submitted on 3 Oct 2019

HAL is a multi-disciplinary open access archive for the deposit and dissemination of scientific research documents, whether they are published or not. The documents may come from teaching and research institutions in France or abroad, or from public or private research centers.
L'archive ouverte pluridisciplinaire HAL, est destinée au dépôt et à la diffusion de documents scientifiques de niveau recherche, publiés ou non, émanant des établissements d'enseignement et de recherche français ou étrangers, des laboratoires publics ou privés. 


\title{
CHRONIQUE
}

\author{
Autour de Cluny
}

\section{CLUNIACENSIA BIBLIOGRAPHICA MINIMA*}

Les considérations qui vont suivre sur l'état de la bibliographie clunisienne nécessitent quelques commentaires préliminaires en guise de mise au point et d'avertissement. Elles n'ont aucune prétention à l'exhaustivité et ne visent pas davantage à offrir l'équivalent du travail fondateur qu'avaient été les Études clunisiennes dans tous leurs états parues ici même il y a quelques années ${ }^{1}$. En effet, en ces temps de communication numérique et d'internationalisation foudroyante des cadres habituels de la recherche, toute bibliographie imprimée est condamnée à l'obsolescence dès sa parution, tout comme un ordinateur l'est, fondamentalement, à la date de son achat. Ce serait, de plus, faire fi des instruments qui existent en ligne, régulièrement et efficacement entretenus : ainsi, la déjà classique Bibliotheca Cluniacensis novissima de l'université de Münster ${ }^{2}$. Délibérément, le parcours ici proposé à partir d'un certain nombre des travaux et publications de ces dernières années offre un aperçu orienté du chemin qu'ont pris les travaux sur Cluny, filon important de l'historiographie française et internationale de ces dernières années. Bien évidemment, tout travail de ce genre est condamné à ètre, s'il n'est pas pur recensement, très personnel et subjectif ; il le sera donc de manière consciente et assumée.

Un autre facteur doit être pris en compte : parler de "Cluny » de manière générale revient à traiter d'une abbaye certes, mais aussi de son réseau monastique, puis d'un ordre à l'extension européenne - et l'implication sociale, religieuse et politique des moines noirs expose au risque d'étendre considérablement le chapitre de la bibliographie clunisienne consacré au " contexte ». C'est dire que, de la synthèse d'ensemble à l'étude de cas sur un prieuré et ses bâtiments, de l'analyse ecclésiologique à la coupe codicologique d'un manuscrit unique, les angles d'approche et les niveaux de réflexion sont multiples - et tout aussi abondants et protéiformes que les publications qui en découlent et dont il ne pourra être rendu fidèlement compte ici. Répétons-le : notre intention n'est pas de produire un recensement bibliographique, ni une sorte de tableau d'honneur proposant le classement implicite ou explicite des publications, mais de mener une simple réflexion personnelle, appuyée sur la bibliographie de la dizaine d'années qui vient de s'écouler.

Les Ateliers clunisiens animés par Dominique Iogna-Prat ont joué un rôle majeur d'entrainement et de structuration des recherches, formant ainsi un groupe, des

* J'adresse mes remerciements à Isabelle Cochelin et Florent Cygler pour leurs relectures et leurs conseils.

1. Dominique Iogna-Prat et Christian SAPIN, «Les études clunisiennes dans tous leurs états : rencontres de Cluny, 21-22 septembre 1993 ", Revue Mabillon, t. 66, 1994, p. 233-265, à compléter par D. Iogna-Prat, "Bibliographie clunisienne (1993-1999) ", Revue Mabillon, t. 72,2000 , p. 269-277.

2. http://www.uni-muenster.de/Fruehmittelalter/fr/Projekte/Cluny/BiblClun/index.html, consulté le 10 mars 2011. Une consultation au 6 octobre, date de dernière relecture de ces lignes, indiquerait sans doute des publications qui n'ont pu y être prises en compte, ainsi, le dernier numéro du Bulletin du Centre d'Études Médiévales d'Auxerre, t. 15, 2011, http://cem.revues. $\mathrm{org} /$ index 11848.html.

Revue Mabillon, n.s., t. 22 (= t. 83), 2011. 
groupes peut-être, de chercheurs qui, pour être informels, n'en ont pas moins été d'une rare fécondité. N'est-ce pas d'ailleurs le caractère résolument peu institutionnalisé de telles rencontres qui a permis cette structuration, ce qui n'est paradoxal qu'en apparence ? Sans nul doute, ces ateliers ont fourni tant un point de concrétion et de rencontre qu'une base de travail intellectuelle et matérielle à la recherche, et pas seulement à la recherche française, sur Cluny. C'est donc aussi de leur héritage qu'il faut parler aujourd'hui. En outre, il convient de souligner, dans les recherches sur Cluny, le rôle du Centre d'Études Médiévales d'Auxerre et du laboratoire du CNRS dont il fait partie, l'ArteHis/UMR 5594 du CNRS. Hors de France, il importe d'observer le rôle appréciable qu'ont joué, dans les universités de Münster et de Dresde, les Sonderforschungsbereiche 231 et 537, respectivement intitulés Pragmatische Schriftlichkeit im Mittelalter (de 1986 à 1999) et Institutionalität und Geschichtlichkeit (de 1997 à 2008) - tout en admettant, pour Cluny, la moindre importance du second programme.

Au tout premier abord, l'impression qui ressort du contact avec l'ensemble des travaux de ces dernières années relatifs à Cluny est, certes, celle d'un relatif ralentissement, voire d'un essoufflement. Ceci tient à plusieurs facteurs. Tout d'abord, l'arrêt ou du moins l'irrégularité des rencontres des Ateliers clunisiens ${ }^{3}$ y a contribué, en particulier pour les recherches menées en France. Ensuite, d'autres centres historiques de la recherche clunisienne ont connu des évolutions institutionnelles défavorables à cette dernière; le départ à la retraite de Joachim Wollasch ${ }^{4}$ a induit un déplacement des centres d'intérêt au regard de ceux qui avaient été les siens à Münster ${ }^{5}$, même si la continuité y est illustrée par les travaux de Franz Neiske ${ }^{6}$ et Maria Hillebrandt ${ }^{7}$, par exemple leur récente contribution au beau volume dirigé par Neil Stratford, dont il sera question plus bas. D'autres chercheurs naguère très engagés dans des recherches ouvertement "clunisiennes" ont fait évoluer leurs centres d'intérêt - en France, D. Iogna-Prat en est un bon exemple, en Allemagne, l'on pourrait citer Gert Melville ${ }^{8}$. Le premier a élargi et modifié son champ d'inves-

3. Un atelier a eu lieu en 2006 à Souvigny après une interruption de quelques années, voir le compte rendu qui en a été fait dans le Bulletin du Centre d'Études Médiévales d'Auxerre: Pascale Chevalier, Arlette Maquet et Ch. Sapin, "Atelier clunisien (compte rendu) ", Bulletin du Centre d'Études Médiévales d'Auxerre [en ligne], t. 10, 2006, mis en ligne le 8 septembre 2006, consulté le 10 mars 2011, http://cem.revues.org/index386.html.

4. Outre le classique Joachim Wollasch, Cluny, "Licht der Welt". Aufstieg und Niedergang der klösterlichen Gemeinschaft (Zurich-Düsseldorf, 1996), il convient de signaler la parution des recueils Wege der Erinnerung im und an das Mittelalter : Festschrift für Joachim Wollasch zum 80. Geburtstag, Andreas SoHn éd., (Bochum, 2011 [Aufbrüche, 3]) et Wege zur Erforschung der Erinnerungskultur : ausgewählte Aufsätze von Joachim Wollasch, Mechthild Sandmann, Angelus A. Häubling, Mechthild Black-Veldtrup éds. (Münster, 2011 [Beiträge zur Geschichte des alten Mönchtums und des Benediktinertums, 47]).

5. Les projets clunisiens de l'Institut für Frühmittelalterforschung sont présentés sur le site de l'université : http://www.uni-muenster.de/Fruehmittelalter/fr/Projekte/Cluny/index.html, consulté le 10 mars 2011.

6. Un exemple : Franz NeIske, "Pro defunctis exorare ut a peccatis solvantur. Theologie und Praxis der Memoria in Cluny ", dans Bücher des Lebens, lebendige Bücher, Peter ErHart et Jakob Kuratlı Hüeblin éds., Saint-Gall, 2010, p. 190-199.

7. Par exemple : Maria Hillebrandt, «Zu Gast in Klöstern. Gastfreundschaft in Hirsau und Cluny ", dans Landkreis Calw. Ein Jahrbuch, t. 25, 2007, p. 215-228.

8. On lui doit l'idée de "Cluny après Cluny ", cf. Gert Melville, "Cluny après "Cluny". Le treizième siècle, un champ de recherches ", Francia, t. 17/1, 1990, p. 91-124. Le même sujet est développé dans son article sur la réforme de Cluny : ID., "Die cluniazensische Reformatio tam in capite quam in membris. Institutioneller Wandel zwischen Anpassung und Bewahrung ", dans Sozialer Wandel im Mittelalter, Wahrnehmungsformen, Erklärungsmuster, Regelungsmuster, Jürgen Miethke et Klaus Schreiner éds., Sigmaringen, 1994, p. 249-297. Au sujet de ses travaux sur les évolutions clunisiennes et de leurs résultats, voir également G. Melville, «Cluny und das französische Königtum. Von "Freiheit ohne Schutz" zu Schutz ohne Freiheit ", dans Die 
tigation ${ }^{9}$ pour publier un grand-œuvre sur la "Maison Dieu " ${ }^{10}$, il s'est intéressé aux concepts d'individu, individualité et individuation ${ }^{11}$ et il ouvre désormais d'amples perspectives du côté de l'ecclésiologie, du christianisme ou de la sociologie des religions ; le second s'est plutôt orienté, d'une part vers une histoire plus générale, comparée et structurelle des ordres religieux ${ }^{12}$ et d'autre part, vers l'historiographie médiévale ${ }^{13}$. De tels exemples invitent, du reste, à prendre en compte la diversification des sujets de recherche et à mettre en garde contre la maladresse qui consisterait à évaluer l'état des recherches à la seule aune du nombre de publications concernant l'abbaye bourguignonne ou des institutions qui porteraient "Cluny » dans leur nom.

Quelles sont donc les évolutions perceptibles dans les travaux récents? Tout d'abord, bien entendu, l'influence des célébrations de 2010 se fait sentir : si nombre de leurs apports attendent encore de passer de la forme orale à la forme écrite, quelques ouvrages ont déjà vu le jour. Le volume publié par N. Stratford, Cluny, onze siècles de rayonnement, par exemple, réunit de nombreux spécialistes dans un ouvrage prévu pour intéresser un large public et où prédominent, sans exclusive toutefois, des sujets d'archéologie et d'histoire de l'art ${ }^{14}$. De tels choix le rendent tout à fait représentatif de ce que l'on pourrait appeler le "mouvement clunisien » de ces quinze à vingt dernières années : il se veut interdisciplinaire, international, ouvert autant que possible. N. Stratford lui-même s'interroge d'emblée sur la pertinence de donner un caractère " clunisien " à un phénomène, ici, à l'art ${ }^{15}$. L'on se souvient des réflexions de D. Iogna-Prat sur le concept de "lettres clunisiennes », par exemple ${ }^{16}$. Cela pourrait bien être, paradoxalement, l'une des caractéristiques fortement partagées des écrits de ces dernières années sur Cluny, ses établissements, son réseau, son ordre : un questionnement permanent sur les catégories employées, jusqu'à la qualification de « clunisien ».

Cette remise en question ne concerne pas les seules catégories heuristiques de l'historiographie moderne, elle vaut aussi pour des catégories transmises ou suggérées de manière plus ou moins erratique par les sources primaires et par les traditions historiographiques aujourd'hui entrées dans notre culture. C'est tout particulièrement sensible dans l'une des publications les plus remarquables de ces dernières

Cluniazenser in ihrem politisch-sozialen Umfeld, Giles Constable, G. Melville et Jörg Oberste éds., Münster, 1998 (Vita regularis. Abhandlungen, 7), p. 405-468.

9. Il suffira ici de citer, dans sa production "clunisienne", D. IoGna-Prat, Ordonner et exclure: Cluny et la société chrétienne face à l'hérésie, au judä̈sme et à l'Islam, 1000-1150, Paris, 1998 (rééd. Paris, 2004).

10. D. Iogna-Prat, La Maison Dieu. Une histoire monumentale de l'Église au Moyen Âge (v. 800-v. 1200), Paris, 2006 (L'univers historique).

11. L'individu au Moyen Âge: individuation et individualisation avant la modernité, D. Iogna-Prat et Brigitte-Miriam Bedos-RezaK éds., Paris, 2005.

12. Voir par ex. Mittelalterliche Orden und Klöster im Vergleich, methodische Ansätze und Perspektiven, G. Melville et Anne Müller éds., Münster, 2007 (Vita regularis. Abhandlungen, 34). Notons que lui aussi a inscrit l'individu dans ses champs de recherche, cf. Das Eigene und das Ganze: zum Individuellen im mittelalterlichen Mönchtum, G. Melville et Markus Schürer éds., Münster, 2002 (Vita regularis. Abhandlungen, 16).

13. Ainsi G. Melville, "Durch Fiktionen von der Wirklichkeit zur Wahrheit : zum mittelalterlichen Umgang mit Widersprüchen zwischen Empirie und kultureller Axiomatik », dans Filition und Fiktionalität in den Literaturen des Mittelalters, Jan-Dirk Müller zum 65. Geburtstag, Ursula Peters et Rainer WARning éds., Munich, 2009, p. 83-104.

14. Cluny 910-2010. Onze siècles de rayonnement, Neil Stratford éd., Paris, 2010.

15. N. Stratford, "Existe-t-il un art clunisien?", dans Cluny 910-2010, op. cit., p. vi-XIII.

16. Elles sont aisément accessibles dans D. Iogna-PRAT, art. "Cluny », dans Dictionnaire des lettres françaises, le Moyen Age, Geneviève Hasenohr et Michel Zink dirs., Paris, 1992, p. $311-316$. 
années : la thèse d'Isabelle Rosé sur Odon de Cluny ${ }^{17}$. Cette dernière a, pour ainsi dire, su intégrer à sa démarche les acquis méthodologiques du "mouvement clunisien " de ces dernières années. Son objectif s'inscrit clairement dans le cadre plus large de la réévaluation et de la réélaboration du genre biographique propre aux dernières décennies. L'évocation d'un "itinéraire biographique " précède donc dans ce livre l'examen des conceptions sociales, religieuses et ecclésiologiques de l'abbé. Tant l'exercice choisi que son objet ou l'époque concernée étaient susceptibles de susciter les difficultés et les problèmes de méthode. C'est là que les notions de dossier, de stratigraphie, d'écriture et de réécriture hagiographique ou documentaire se montrent utiles et pertinentes, en permettant une approche toujours active et critique des sujets abordés. L'interdisciplinarité résolument pratiquée s'y est avérée tout aussi féconde : ce ne sont pas seulement différents genres textuels, avec leur méthodologie, qui sont exploités, mais aussi les résultats de l'archéologie. Telle était déjà, du reste, l'une des caractéristiques de l'ouvrage publié par Didier Méhu il y a quelques années ${ }^{18}$. L'attention portée par I. Rosé aux phénomènes de construction du social, comme l'annonce d'emblée le titre de son livre, prend également place dans toute une tradition d'exploitation des textes, notamment ecclésiologiques, caractéristique des productions de ces dernières années.

À l'autre bout de la chaîne de l'histoire clunisienne, des lacunes criantes continuent d'affecter nos connaissances sur Cluny aux temps modernes, et en fait, déjà à partir du XIV ${ }^{\mathrm{e}}$ siècle. Les travaux de Giles Constable ${ }^{19}$ et des " écoles " de Münster, puis de Dresde ${ }^{20}$, avaient avec d'autres contribué à enrichir l'histoire des XII et XIII siè$\operatorname{cles}^{21}$. Les travaux de Denyse Riche ${ }^{22}$ ont aidé à voir plus clair dans les deux siècles suivants ; et un livre récemment paru, sous sa plume et celle de Daniel-Odon Hurel, a tenté le pari de brosser à nouveaux frais un tableau de Cluny, de sa congrégation puis de son ordre du $\mathrm{x}^{\mathrm{e}}$ au xviII ${ }^{\mathrm{e}}$ siècle, en intégrant autant que possible les données de la recherche récente ${ }^{23}$. Il n'est, du reste, que de voir les notes bibliographiques correspondant à chaque chapitre pour constater que la situation historiographique est toujours assez déséquilibrée en faveur du Moyen Âge, et du Moyen Âge central notamment. L'histoire des religieux et de leurs ordres aux XVI ${ }^{\mathrm{e}}$-XVIII ${ }^{\mathrm{e}}$ siècles est en règle générale bien mal connue ; pourquoi en irait-il autrement de Cluny ${ }^{24}$ ? Le choix

17. Isabelle Rosé, Construire une société seigneuriale, itinéraire et ecclésiologie de l'abbé Odon de Cluny (fin du IXe-milieu du X $X^{e}$ siècle), Turnhout, 2008 (Collection d'études médiévales de Nice, 8).

18. Didier Ménu, Paix et communautés autour de l'abbaye de Cluny ( $X^{e}-X V^{e}$ siècle), Lyon, 2001 (Collection d'histoire et d'archéologie médiévales, 9).

19. Outre les textes repris dans le recueil cité infra n. 27, on rappellera ici, au sein d'une abondante bibliographie, ses éditions, par exemple Statuta Petri Venerabilis Abbatis Cluniacensis IX (1146/7), G. Constable éd., dans Consuetudines Benedictinae Variae (Saec. XSaec. XIV), Siegburg, 1975 (Corpus Consuetudinum Monasticarum, 6), p. 19-106.

20. Les travaux émanant de ces équipes de recherche et dont le contenu dépasse largement Cluny ont été présentés dans G. Melville et Florent Cygler, « Nouvelles approches historiographiques des ordres religieux en Allemagne. Le groupe de recherches de Dresde sur les structures institutionnelles des ordres religieux au Moyen Âge ", Revue Mabillon, t. 73, 2001, p. 314-321.

21. Mème sur ces terrains mieux balisés, beaucoup reste à faire, comme le démontre par exemple la thèse de Marc P. Saurette sur Pierre le Vénérable et son article: "Thoughts on Friendship in the Letters of Peter the Venerable ", Revue Bénédictine, t. 120, 2010, p. 321-346.

22. Denyse Riche, L'ordre de Cluny à la fin du Moyen Áge : le "vieux pays clunisien", $X I I^{e}-X V^{e}$ siècle, Saint-Étienne, 2000 (CERCOR. Travaux et recherches, 13).

23. Daniel-Odon Hurel et D. Riche, Cluny, de l'abbaye à l'ordre clunisien, $X^{e}$. XVIII siècle, Paris, 2010.

24. Parmi les travaux récents qui contribuent à remédier à cet état de fait, cf. Grégory Goudot, «Monachisme clunisien et vie rurale sous l'Ancien Régime. Le cas auvergnat de Menat aux XVII et XVII" siècles", dans Histoire et sociétés rurales, t. 25, 2006, p. 9-35 ; ID., "Le personnel de l'ordre de Cluny en France à la veille de la Révolution. Sources, méthodes et 
des deux auteurs s'est du moins porté vers la présentation d'un tableau à la fois général, approfondi et accessible. Tout en reconnaissant que cela a pu les mener à des tris drastiques dans la bibliographie, à dominante nettement française à quelques exceptions près, il n'en demeure pas moins que ce livre est le bienvenu.

Il révèle d'ailleurs un certain besoin de bilan, ou, tout au moins, de respiration allant de pair avec le tout relatif ralentissement des publications. La vitalité des activités de commémoration en procède aussi. On constate aussi, au cours de ces dernières années, un regain des publications consacrées à des tours d'horizon de travaux plus anciens. Les rééditions et collections d'articles trouvent leur raison d'être dans la volonté de rendre hommage aux mérites scientifiques des personnes concernées et de leur œuvre, qu'il s'agisse des Études clunisiennes de D. Iogna-Prat aux "Médiévistes français " ${ }^{25}$, ou des volumes de Giancarlo Andenna ${ }^{26}$ et de G. Constable ${ }^{27}$ pour "Vita regularis". Là encore, du reste, le champ des travaux dépasse largement Cluny : que l'on songe aux bilans historiographiques généraux parus en diverses langues, tels Dove va la storiografia monastica en Europa ${ }^{28}$, ou Tendances actuelles de l'histoire du Moyen Âge en France et en Allemagne ${ }^{29}$, ou encore, dans un numéro spécial des Cahiers de civilisation médiévale, en 2006, la suite d'articles sur La médiévistique au $X X^{e}$ siècle : bilan et perspectives. À cela s'ajoutent d'autres "bilans et perspectives ", tantôt sous la forme d'une œuvre collective patronnée par une institution ${ }^{30}$, et tantôt sous la forme d'essais plus personnels, comme ceux d'Alain Guerreau ${ }^{31}$ ou de Jacques Heers ${ }^{32}$ pour citer deux options radicalement différentes ${ }^{33}$. Au regard de ces diverses publications, l'histoire clunisienne stricto sensu fait-elle autre chose que décliner sa propre version des interrogations et des retours en arrière largement pratiqués par les historiens de ces dernières années, et parmi eux, par les médiévistes?

L'un des gains de l'historiographie clunisienne est probablement son internationalisation, ou, plus exactement, l'attention accrue que les historiens des différentes aires géographiques prêtent à leurs activités respectives. L'Italie présente une riche tradition en matière de recherche clunisienne, dont Cinzio Violante était l'un des repré-

premiers résultats d'une enquête ", dans Regards sur les sources. Histoire moderne et contemporaine, Jean-Claude Caron dir., Clermont-Ferrand, 2005, p. 25-40 ; ID., " Pour une histoire de l'Ecclesia cluniacensis à l'époque moderne", Cahiers clunisiens, t. 1, 2007, p. 28-31. Pour sa part, l'auteur de ces lignes s'était essayé à une perspective de longue durée dans Sébastien Barret, La mémoire et l'écrit : l'abbaye de Cluny et ses archives, X'e-XVIII siècle, Münster, 2004 (Vita regularis. Abhandlungen, 19).

25. D. Iogna-Prat, Études clunisiennes, Paris, 2002 (Les médiévistes français, 2).

26. Giancarlo Andenna, Sanctimoniales Cluniacenses, studi sui monasteri femminili di Cluny e sulla loto legislazione in Lombardia (XI-XV secolo), Münster, 2004 (Vita regularis. Abhandlungen, 20).

27. G. Cionstable, The Abbey of Cluny, a Collection of Essays to Mark the ElevenHundredth Anniversary of its Foundation, Berlin, 2010 (Vita regularis. Abhandlungen, 43).

28. Dove va la storiografia monastica in Europa? Temi e metodi di ricerca per lo studio della vita monastica e regolare in età medievale alle soglie del terzo millennio. Atti del Convegno internazionale, Brescia-Rodengo, 23-25 marzo 2000, G. Andenna éd., Milan, 2001.

29. Les tendances actuelles de l'histoire médiévale en France et en Allemagne, Jean-Claude Schnitt et Otto Gerhard Oexle dirs., Paris, 2002.

30. Les historiens français à l'œuvre, 1995-2010, Jean-François Sirinelli, Pascal Cauchy et Claude Gauvard dirs., Paris, 2010, sous les auspices du CTHS.

31. Alain Guerreau, L'avenir d'un passé incertain. Quelle histoire du Moyen Âge au $X X I^{e}$ siècle?, Paris, 2001.

32. Jacques Heers, Le Moyen Age, une imposture, Paris, 2008 (Tempus, 243), réédition d'un ouvrage initialement paru en 1992.

33. Plus récemment aussi, une troisième voie est empruntée par Patrick Boucheron, Faire profession d'historien, Paris, 2010. 
sentants les plus notoires ${ }^{34}$. Cluny et ses établissements ont toujours fait partie des travaux italiens sur les ordres religieux, pas forcément toujours en eux-mêmes, mais du moins comme les éléments d'un plus vaste panorama. Il convient de mentionner à nouveau dans cette perspective les travaux de G. Andenna, à la fois inscrits dans la tradition déjà ancienne des études à orientation régionale, en ce cas sur la Lombardie, et ouverts au secteur assez peu exploré du monachisme féminin, en l'occurrence par des recherches sur le monachisme féminin d'inspiration clunisienne ${ }^{35}$. Ce même complexe d'approche régionale et de réflexion, d'une part sur l'ecclésiologie et l'identité, d'autre part sur l'historiographie et la méthodologie, est au cœur des travaux récents ou plus anciens de Glauco Maria Cantarella ${ }^{36}$. La Péninsule ibérique apporte aussi son lot d'études et de publications, outre l'implication de ses chercheurs dans certains projets de recherche d'ampleur européenne - l'historiographie est, du reste, en ce domaine d'autant plus intéressante qu'elle a pu considérer Cluny comme une sorte de tête de pont de voisins français extrêmement envahissants ${ }^{37}$. Les travaux des chercheurs espagnols relatifs à un certain nombre de prieurés ont permis de faire avancer sensiblement la réflexion et de rendre accessible de nouvelles documentations, ainsi sur Carrión de Los Condes ${ }^{38}$, San Isidro de Dueñas ${ }^{39}$, Sahagún ${ }^{40} \ldots$ jusqu'à la synthèse parue sous la plume de Carlos Manuel Reglero de La Fuente ${ }^{41}$.

De telles approches régionales ne se limitent pas, bien entendu, au Sud du continent européen. La présence clunisienne en terre germanique et les relations des clunisiens avec les empereurs, outre le sujet d'un débat de plusieurs années entre J. Wollasch et Karl Josef Benz ${ }^{42}$, a ainsi fourni la matière d'études comme celle de

34. Citons pour mémoire les publications déjà anciennes, mais devenues classiques, de son recueil "Per una riconsiderazione della presenza cluniacense in Lombardia », dans Cluny in Lombardia. Atti del convegno storico celebrativo del IX centenario della fondazione del priorato cluniacense di Pontida, 22-25 aprile 1977, 2 vol., Césène, 1979-1981 (Italia benedettina, 1).

35. Voir dans Sanctimoniales cluniacenses, op. cit., ses considérations sur le monachisme féminin d'obédience clunisienne, aux p. 1-48, et la riche bibliographie donnée par l'ouvrage.

36. Il est récemment revenu sur une question jamais résolue et toujours renaissante : Glauco

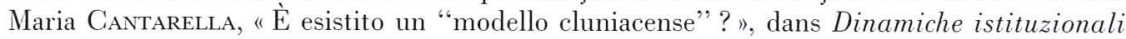
delle reti monastiche e canonicali nell'Italia dei secoli X-XII, Fonte Avellana, 29-31 agosto 2006, Nicolangelo D'Acunto éd., Negarine di S. Pietro in Cariano (Verona), 2007, p. 61-85 ; outre de nombreux travaux sur Cluny et les clunisiens en Lombardie, on lui doit la synthèse $I$ monachi di Cluny, parue en 1993, au sein d'une riche bibliographie qui ne concerne pas que Cluny.

37. Patrick Henriet, «Moines envahisseurs ou moines civilisateurs? Cluny dans l'historiographie espagnole, xnI $^{\mathrm{e}}-\mathrm{xx}^{\mathrm{e}}$ siècles ", Revue Mabillon, t. 72, 2000, p. 135-159.

38. José Luis Senra Gabriel y Galán, "La puerta como dogma : a propósito de un nuevo descubrimiento en la iglesia románica de San Zoilo de Carrión de los Condes (Palencia) ", Archivo Español de Arte, t. 81, 2008, p. 139-150 ; Fr. Neiske et Carlos Manuel Regr fro de LA Fuente, «Das neu entdeckte Necrolog von San Zoilo de Carrión de los Condes. Ein Beitrag zum Totengedenken der Abtei Cluny ", Frühmittelalterliche Studien, t. 41, 2007, p. 141-184.

39. C. M. Reglero de la Fuente, El monasterio de San Isidro de Dueñas en la Edad Media. Un priorato cluniacense hispano (911-1478). Estudio y collección documental, León, 2005 (Fuentes y estudios de historia leonesa, 106).

40. C. M. Reglero de la Fuente, "La primera reforma cluniacense de Sahagún, el concilio de Burgos y la Crisis de 1080. Revisión cronológica y desarrollo ", dans Monarquía y sociedad en el Reino de León. De Alfonso III a Alfonso VII, León, 2007 (Fuentes y estudios de historia leonesa, 118), t. II, p. 689-732, ou encore Javier PÉrez Gil et Javier Rivera Blanco, « Sahagún y Cluny. Vidas paralelas ", dans Los grandes monasterios benedictinos hispanos de época romanica (1050-1200), Aguilar de Campo, 2007, p. 87-119.

41. C. M. Reglero de La Fuente, Cluny en España. Los prioratos de la provincia y sus redes sociales (1073-ca. 1270), León, 2008 (Fuentes y estudios de historia leonesa, 122).

42. Par exemple: J. Wollasch, "Kaiser Heinrich II. in Cluny ", Frühmittelalterliche Studien, t. 3, 1969, p. 327-342 ; Karl-Josef Benz, "Kaiser Heinrich II. in Cluny?», Frühmittelalterliche Studien, t. 8, 1974, p. 155-178 ; ID., "Heinrich II. und Cluny », Revue Bénédictine, 
Florian Lamke, Cluniacenser am Oberrhein ${ }^{43}$. En France, les travaux à orientation régionale, dont Eliana Magnani avait déjà donné un excellent exemple pas seulement clunisien ${ }^{44}$, sont également représentés, entre autres, par la thèse d'Arlette Maquet portant sur l'Auvergne ${ }^{45}$. Les territoires des Flandres constituent l'espace privilégié des recherches de Steven Vanderputten ${ }^{46}$. Bref, après l'avancée réalisée par les travaux de Dietrich Poeck donnant un aperçu général de l'Ecclesia Cluniacensis ${ }^{47}$, la couverture régionale obtenue par diverses études récentes attentives aux réalités locales progresse, ce qui est une excellente chose - et du reste, l'observation ne vaut pas seulement pour les clunisiens, mais tout autant pour les franciscains, par exemple ${ }^{48}$, ou encore pour les dominicains grâce aux publications de Simon Tugwell ${ }^{49}$. Là encore, il est permis de se demander si, au fond, une partie des études clunisiennes ne doit pas être inscrite dans le contexte plus général de l'histoire des ordres et congrégations religieuses, tant il est vrai que, ces dernières années, la constitution et le fonctionnement des réseaux régionaux et des provinces - quand il y en a - ont été mis au cœur de bien des recherches et ont révélé bien des complexités ${ }^{50}$.

Un secteur important de la recherche concerne la mise à disposition de sources primaires, tout comme la critique et les réflexions méthodologiques que leur étude suscite. Dans ce registre, notons tout d'abord, parmi la publication d'un certain nombre de textes, celle de la Vie de Géraud d'Aurillac par Odon, document important et souvent exploité par les historiens de l'abbaye et de la société - parmi lesquels I. Rosé, déjà citée, ou Isabelle Cochelin ${ }^{51}$. Mais si cette dernière a encore dû s'appuyer sur le texte donné par la Patrologie latine, qui reprenait en fait une édition

t. 84, 1974, p. 313-337 ou ID., " À propos du dernier voyage de l'impératrice Adélaïde en 999 ", Revue d'histoire ecclésiastique, t. 67, 1972, p. 81-91, aux p. 84-85, suivis des réponses de J. Wollasch, "Cluny und Deutschland", Studien und Mitteilungen zur Geschichte des Benedilitinerordens und seiner Zweige, t. 103, 1992, p. 7-32, aux p. 12, n. 39 et 16, n. 55.

43. Florian Lamke, Cluniacenser am Oberrhein. Konfliktlösungen und adlige Gruppenbildung in der Zeit des Investiturstreits, Fribourg/Brisgau-Munich, 2009 (Forschungen zur oberrheinischen Landesgeschichte, 54).

44. Eliana Magnani, Monastères et aristocratie en Provence (milieu X'e-début XII siècle), Münster, 1999 (Vita regularis. Abhandlungen, 10).

45. Voir la présentation de sa thèse dans Bulletin du Centre d'Études Médiévales d'Auxerre, t. 11, 2007, en ligne: http://cem.revues.org/index2142.html, consulté le 13 mars 2011.

46. Steven Vanderputten, "A time of great confusion. Second-generation Cluniac reformers and resistance to centralization in the county of Flanders (c. 1125-45) ", Revue d'histoire ecclésiastique, t. 102, 2007, p. 47-75 ; ID., «Fulcard's pigsty : Cluniac reformers, dispute settlement, and the lower aristocracy in early twelfth-century Flanders ", in Viator. Medieval and Renaissance Studies, t. 38, 2007, p. 91-115; et à propos d'Anchin, ID., "A Compromised Inheritance. Monastic Discourse and the Politics of Property Exchange in Early-TwelfthCentury Flanders ", The Journal of Ecclesiastical History, t. 61, 2010, p. 229-251.

47. Dietrich W. Poeck, Cluniacensis Ecclesia. Der cluniacensische Klosterverband (10.12. Jahrhundert), Munich, 1998 (Münstersche Mittelalter-Schriften, 71).

48. Jens Röhrkasten est l'un de ceux qui font progresser ces études, voir par exemple Franciscan Organisation in the Mendicant Context : Formal and Informal Structures of the Friars' Lives and Ministry in the Middle Ages, J. Röhкasten et Michael Robson éds., Berlin, 2010 (Vita regularis. Abhandlungen, 44).

49. Un exemple pris parmi les articles très nourris qu'il fait régulièrement paraître dans l'Archivum fratrum Praedicatorum : Simon Tugwell, "The evolution of Dominican structures of government : terminology, nomenclature and ordo of Dominican provinces ", Archivum fratrum Praedicatorum, t. 75, 2005, p. 29-94.

50. Un secteur renouvelé de ce point de vue a été celui de la vie canoniale : cf. Les chanoines réguliers : émergence et expansion (XI -XIII siècles), Michel PARIsse éd., Saint-Étienne, 2009 (CERCOR. Travaux et recherches, 19).

51. Isabelle Cochelin, "Quête de liberté et réécriture des origines : Odon et les portraits corrigés de Baume, Géraud et Guillaume ", dans Guerriers et moines : conversion et sainteté aristocratique dans l'Occident médiéval, Michel Lauwers éd., Antibes, 2002, p. 183-215. 
antérieure dans la Bibliotheca Cluniacensis ${ }^{52}$, les historiens disposent maintenant de l'édition critique d'Anne-Marie Bultot-Verleysen, accompagnée d'une traduction en regard et complétée par une riche introduction historique, littéraire et ecdotique ${ }^{53}$. C'est selon les mêmes modalités qu'elle avait déjà édité des Miracula de saint Géraud quelques années auparavant ${ }^{54}$. Tout comme dans le cas de l'édition récente de la Vita de Guillaume de Volpiano ${ }^{55}$, la traduction devient de plus en plus fréquente désormais, à mesure que s'apaisent les débats sur la pertinence d'une telle démarche. Parmi les travaux les plus récents, signalons la soutenance, en mars 2011, d'une thèse de l'École des chartes dont l'auteur, Franz Dolveck, a procédé à l'édition critique des œuvres poétiques de Pierre le Vénérable et Pierre de Poitiers, ainsi qu'à celle du Mariale de Bernard de Morlas - la publication des positions de thèses ${ }^{56}$ permettant d'en faire mention ici et les éloges du jury d'en attendre de prochaines publications. Dans le domaine des textes de nature diplomatique, on se gardera d'oublier l'entreprise, encore en cours, de la publication avec fac-similés des plus anciens documents originaux de l'abbaye, dans la Series Gallica des Monumenta Palaeographica Medii Aevi ${ }^{57}$, la mine représentée par les chartes « clunisiennes » n'ayant jamais cessé d'être exploitée depuis les grands travaux d'André Déléage et Georges Duby, par exemple par Barbara H. Rosenwein ${ }^{58}$.

Mais il faut aussi évoquer tout ce qui se fait dans le domaine des nouvelles technologies. La mise en ligne des chartes clunisiennes a été engagée par les soins de l'université de Münster ${ }^{59}$, sur la base de leur publication par Auguste Bernard et Alexandre Bruel ${ }^{60}$, tandis qu'elles figurent aussi dans le corpus des Chartae Burgundiae Medii Aevi de l'ArteHis de Dijon ${ }^{61}$. Par ce dernier biais, les chartes clunisien-

52. Bibliotheca Cluniacensis in qua SS. Patrum abbatum Cluniacensium vitae, miracula, scripta, statuta, privilegia..., Martin Marrier et André Duchesne éds., Paris, 1614 (rééd. Màcon, 1915).

53. Odon de Cluny. Vita sancti Geraldi Auriliacensis, éd. et trad. Anne-Marie BultotVerleysen, Bruxelles, 2009 (Subsidia Hagiographica, 89).

54. A.-M. Bultot-Verleysen, "Des Miracula inédits de saint Géraud d'Aurillac († 909). Étude, édition critique et traduction française ", Analecta Bollandiana, t. 118, 2000, p. 47141.

55. "Guillaume de Volpiano. Un réformateur en son temps (962-1031). Vita domni Willelmi de Raoul Glaber, texte, traduction, commentaire », Véronique GAzeau et Monique Goullet éds., Caen, 2008.

56. Franz Dolveck, «Poétique de louange et d'éloge à Cluny au xiı siècle : édition critique des œuvres poétiques complètes de Pierre le Vénérable et de Pierre de Poitiers et du Mariale de Bernard de Morlas ", dans École nationale des chartes, Positions des thèses... des élèves de la promotion 2011, Paris, 2011, en ligne : http://theses.enc.sorbonne.fr/2011/dolveck, consulté le 11 mars 2011.

57. Les plus anciens documents originaux de l'abbaye de Cluny, éd. Hartmut Atsma, S. BArret et Jean Vezin, Turnhout, 1997-2002, 3 vol. parus (Monumenta Palaeographica Medii Aevi. Series Gallica).

58. Après la publication de son important ouvrage To Be the Neighbour of Saint Peter : The Social Meaning of Cluny's Property, $909-1049$ (Ithaca-Londres, 1989), elle a continué à en explorer les multiples facettes, par exemple dans "Circles of Affection in Cluniac Charters », dans Écritures de l'espace social : mélanges d'histoire médiévale offerts à Monique Bourin, Didier Boisseuil, Pierre Chastang, Laurent Feller, Joseph Morsel éds. (Paris, 2010, p. 397. 415), pour ne citer que deux titres au sein d'une œuvre féconde.

59. Tobias Freund, Maria Hillebrandt, Fr. Neiske et Michel Petitjean, Cartae Cluniacenses electronicae : Datenbank der Urkunden der Abtei Cluny (802-1300), http://fruehmittelalter. uni-muenster.de/cce, consulté le 11 mars 2011.

60. Recueil des chartes de l'abbaye de Cluny, Auguste Bernard et Alexandre Bruel éds., Paris, 1876-1903 (Collection de documents inédits sur l'histoire de France) ; l'ouvrage, numérisé par les soins de la Bibliothèque nationale de France, est accessible sur son site Gallica (http://gallica.bnf.fr/, consultation le 15 juin 2011).

61. E. Magnani, Marie-José Gasse-Grandjean et Nicolas Perreaux, Cartae Burgundiae Medii Aevi, http://www.artehis-cbma.eu/, consulté le 11 mars 2011. 
nes entrent dans toute une série d'approches renouvelées par la technique et la statistique. De la sorte, Cluny n'est plus, ou plus forcément, le centre des recherches opérées ; mais par sa position dans l'ensemble des institutions fournissant sources et données, Cluny focalise vivement l'orientation de recherches qui dépassent ce monastère, reprenant et renforçant un rôle qu'il avait déjà tenu dans de célèbres études passées ${ }^{62}$. Ce phénomène s'amplifie si l'on considère, en même temps que Cluny, son environnement : à travers les chartes bourguignonnes, des milliers de documents font ici l'objet, non seulement d'une mise à disposition renouvelée, mais aussi, potentiellement du moins, d'un traitement innovant ${ }^{63}$. À cela s'ajoutent les numérisations en mode image qui permettent elles aussi une mise à disposition pratique de bien des richesses documentaires.

Bien évidemment, les questions méthodologiques que posent ces développements ne se limitent pas à Cluny, mais on ne peut se dispenser de les évoquer ici. Sans doute, ces opérations de dématérialisation permettent la mise à disposition d'une grande quantité de documents et facilitent leur interrogation. On ne doit pas cependant se dissimuler les changements qu'elles induisent dans l'approche même du texte, ce qui était, du reste, déjà sensible lors de l'apparition des premiers instruments numériques il y a quelques années. Permettant de fructueuses approches statistiques, la mise en corpus électroniques peut aussi tendre à diluer dans l'expérience du chercheur la notion de texte fini au profit de l'accès plus abstrait à une sorte de réserve de renseignements ou de données. Non, bien sûr, que ceci soit vraiment neuf : la pêche au renseignement ponctuel dans une documentation sur papier éparpillée peut amener des phénomènes proches - après tout, aborder la Bible par le biais de concordances imprimées n'est pas la même chose que de retrouver les citations d'un texte que l'on connaîtrait par cœur. Mais la taille grandissante des corpus numérisés produit un effet de généralisation qui n'est sûrement pas négligeable. Pour le dire d'une manière un peu caricaturale, les textes sont ainsi plus souples et d'une exploitation plus aisée, mais aussi moins fermement délimités et déterminés. Le traitement de masse opéré à partir de tels corpus, de même que la notion de masse critique autorisant à déclarer la validité des traitements statistiques sont à mettre en balance avec la perte heuristique qu'implique la raréfaction des travaux d'orfèvrerie sur des corpus diplomatiques limités mais explorés en toute profondeur. Le choix est d'autant plus difficile que, si l'on en juge par la distribution des financements, les projets les plus favorisés sont, en règle générale, ceux qui portent sur de grandes quantités de documents. Il sera, du reste, très intéressant de voir quelle direction prendront les éditions de textes "non-diplomatiques" ou d'observer l'éventuelle évolution de la partie clunisienne des Monumenta Palaeographica Medii Aevi. En définitive, dans ce secteur en plein développement, les enjeux ne sont pas seulement la mise à disposition de sources, éditées ou non, et d'études plus ou moins monographiques ou partielles : les modalités de la communication scientifique s'en trouvent également renouvelées, comme elles l'ont été ailleurs. C'est ce que montre bien la parution récente du carnet de recherche Laetus Diaconus conçu pour l'échange et la présentation interdisciplinaires dans l'orbite de l'ArteHis ${ }^{64}$ et hébergé sur une plate-forme fournie à cet effet par Hypotheses.org.

Dans un autre registre, il convient de prendre la mesure de l'avancée des travaux de publication du corpus de la sculpture clunisienne ${ }^{65}$. Ils doivent, à leur tour, être

62. Ainsi, Georges Duby, La société aux XI et XII siècles dans la région mâconnaise, Paris, 1953 ou André DéLÉAge, La vie rurale en Bourgogne jusqu'au début du XI e siècle, Màcon, 1941.

63. Voir à ce sujet les réflexions présentées par Alain Guerreau, «Pour un corpus de textes latins en ligne / Projekt : ein Korpus lateinischer Texte open access on line ", en ligne sur le carnet Laetus diaconus : http://laetusdiaconus.hypotheses.org/540, consulté le 28 avril 2011.

64. Laetus diaconus, éd. par N. Perreaux, http://laetusdiaconus.hypotheses.org/, consulté le 11 mars 2011 .

65. Entreprise dirigée par N. Stratford ; voir N. Stratford, Brigitte Maurice-Chabard et David Walsh, avec les contributions de Annie Blanc, Vincent Droguet, Antoine Paillet et al., 
replacés dans une longue tradition de recherche sur les aspects monumentaux et archéologiques de l'histoire de l'abbaye, tradition dans laquelle le nom de Kenneth J. Conant résonne encore, malgré tous les compléments qui ont pu et dû être apportés à son œuvre pionnière ${ }^{66}$. C'est peut-être bien, du reste, dans le domaine de l'archéologie et de l'histoire de l'art, ou même à la confluence de ces deux disciplines entre elles d'une part, avec la liturgie et l'histoire culturelle d'autre part, que les développements historiographiques des dernières années ont produit les résultats les plus manifestes. Les études d'Anne Baud ${ }^{67}$ ou de Kristina Krüger ${ }^{68}$ se sont toujours voulues résolument polyvalentes, tant dans les sources et les méthodes employées que dans les objectifs poursuivis. Symétriquement, les travaux de Didier Méhu s'appuient aussi sur l'expérience d'archéologue de leur auteur. L'impression du rédacteur de ces lignes est que, d'une certaine manière, les travaux fondés sur l'analyse d'éléments monumentaux, artistiques ou archéologiques se laissent plus profondément renouveler et bouleverser par la prise en compte d'autres secteurs de l'histoire du Moyen Âge que l'inverse, même si ces autres secteurs peuvent trouver dans les apports de l'archéologie et de l'histoire de l'art des éclairages complémentaires parfois importants. Cette fois encore, les renouvellements méthodologiques ne se sont certainement pas exclusivement produits à la faveur des études clunisiennes, même si un certain nombre de problématiques sont spécifiques aux moines noirs ${ }^{69}$. En tout cas, c'est bien ce qui semble se dégager d'un livre comme celui de Carolyn Marino Malone sur Saint-Bénigne de Dijon, au sous-titre évocateur : Interprétation politique, liturgique et théologique ${ }^{70}$. Les recherches récentes et les activités qui sont venues grossir le sillage des commémorations de 2010 ont, là aussi, beaucoup apporté, en relation avec les découvertes faites à la faveur des travaux et aménagements opérés dans les bâtiments conventuels de Cluny ${ }^{71}$. Une simple consultation des derniers Bulletins du Centre d'Études Médiévales d'Auxerre montre du reste que ces activités et ces

et le concours de Nadine Roivé, Gilles Rollier et Jean-Denis SAlvèQue, Corpus de la sculpture de Cluny. Les parties orientales de la Grande Église Cluny III, T. I, vol. 1 et 2, Paris, 2011.

66. Kenneth J. Conant, Cluny, les églises et la maison du chef d'ordre, Mâcon, 1968 ; on se reportera avec profit à la bibliographie des travaux de K. J. Conant établie par Minott KerR, The Bibliography of Kenneth John Conant on Cluny, sur le site de l'université de Münster : http://www.uni-muenster.de/Fruehmittelalter/Projekte/Cluny/BC/bibconant. html, consultéle 11 mars 2011.

67. Anne Baud, Cluny, un grand chantier médiéval au cour de l'Europe, Paris, 2003 ; voir également : EAD., " La chapelle de l'abbé et le passage Galilée à Cluny : les sources graphiques et textuelles à l'épreuve de l'archéologie ", dans Texte et archéologie monumentale : approches de l'architecture médiévale. Actes du colloque international tenu à Avignon du 30 novembre au 2 décembre 2000, Philippe Bernardi éd., Montagnac, 2005, p. 58-65.

68. Kristina KrüGER, Die romanischen Westbauten in Burgund und Cluny. Untersuchungen zur Funktion einer Bauform, Berlin, 2003.

69. Le renouvellement de perspective est manifeste, par exemple, dans certaines études consacrées aux cisterciens, dont Megan CAssidy-Welch, Monastic Spaces and their Meanings : Thirteenth-Century Cistercian English Monasteries, 2001 ; ou encore aux chanoines augustiniens (Sheila BONDE et Clark MAINES, "Ne aliquis extraneus claustrum intret : Entry and Access at the Augustinian Abbey of Saint-Jean-des-Vignes, Soissons " dans Perspectives for an Architecture of Solitude. Essays on Cistercians, Art and Architecture in honour of Peter Ferguson, Terryl N. Kinder éd., Turnhout, 2004 (Medieval Church Studies, 11), p. 173-86) et aux franciscains (G. MelviLle et A. MüLler, «Franziskanische Raumkonzepte : zur symbolischen Bedeutung des inneren und äußeren Hauses ", Revue Mabillon, t. 82, 2010, p. 105-137, en particulier les p. 119-136 dans lesquelles A. Müller traite de l'architecture et du plan des couvents franciscains).

70. Carolyn Marino Malone, Saint-Bénigne de Dijon en l'an mil, Totius Galliae basilicis mirabilior : interprétation politique, liturgique et théologique, Turnhout, 2009 (Disciplina monastica, 5).

71. Voir par exemple A. BAud et Ch. SAPIN, "[Opérations archéologiques 2008-2009] : l'abbaye Saint-Pierre-Saint-Paul de Cluny ", Bulletin du Centre d'Etudes Médiévales 
découvertes sont très loin de se limiter à l'abbaye-mère de Cluny ou à sa congrégation ${ }^{72}$. Dans le domaine de l'image "pure", signalons également le numéro de la revue Art de l'enluminure consacré à Cluny, représentatif de la rencontre entre des recherches au long cours, un large public et une ambiance de célébration ${ }^{73}$.

Un autre complexe extrêmement fructueux s'est développé autour des questions de liturgie et de vie quotidienne - qui, on vient de le voir, sont liées à d'autres secteurs de la recherche et liées entre elles par les sources et par leurs implications communes. I. Cochelin et Susan Boynton illustrent cet apport de manière exemplaire par leurs travaux sur Cluny et ses coutumes ${ }^{74}$. L'étude des coutumiers de Bernard et d'Ulrich comme sources liturgiques, que l'on doit à cette dernière, n'est qu'un exemple dans un riche ensemble de travaux venus s'ajouter à ceux de Michel Huglo ${ }^{75}$, Catherine Bonnin-Magne ${ }^{76}$ ou Manuel Pedro Ramalho Ferreira ${ }^{77}$. Dans ce complexe quelques thèmes traités avec une particulière attention par certains chercheurs prennent un relief nouveau, tels ceux de la mort par Frederick Paxton ${ }^{78}$, des âges et des cycles de la vie par I. Cochelin ${ }^{79}$, du silence et de la communication par Scott Bruce ${ }^{80}$.

Dans la catégorie des publications dans lesquelles Cluny prend place sans en être le sujet principal, mais en fournissant une bonne part de la matière, il faut citer par exemple le livre de Jörg Sonntag sur les aspects symboliques de la vie conventuelle ${ }^{81}$

d'Auxerre, t. 14, 2010, en ligne : http://cem.revues.org/index11498.html, consulté le 13 mars 2011.

72. Voir par exenple Ibid., "[Opération archéologiques 2008-2009]", en ligne : http:// cem.revues.org/index11439.html\#operations-archeologiques-2009-2010, consulté le 13 mars 2011.

73. L'enluminure à l'abbaye de Cluny, numéro thématique de L'art de l'enluminure, t. 33, juin/juillet/août 2010. Un fragment du lectionnaire de Cluny assez récemment acquis par le Musée national du Moyen Âge est visible en ligne sur le site de ce dernier : http://www.museemoyenage.fr/pages/page_id18569_ull2.htm, consulté le 28 avril 2011, commentaire sous http://www.musee-moyenage.fr/documents/Mois2005-01.pdf, même date de consultation.

74. From Dead of Night to End of Day. The medieval customs of Cluny / Du cour de la nuit à la fin du jour. Les coutumes clunisiennes au Moyen Âge, Susan Boynton et Isabelle Cochelin éds., Turnhout, 2005 (Disciplina monastica, 3) et notamment, au sein de ce volume, S. Boynton, "The Customaries of Bernard and Ulrich as Liturgical Sources ", aux p. 109. 130.

75. Un exemple en est donné dans ce volume même de la Revue Mabillon.

76. Un résumé de sa thèse de doctorat, soutenue en 2005, a été publié dans Catherine Bonnin-Magne, "Le sanctoral clunisien ( $\mathrm{x}^{\mathrm{e}}-\mathrm{xv}^{\mathrm{e}}$ siècles), Bulletin du Centre d'Études Médiévales d'Auxerre, t. 10, 2006, en ligne: http://cem.revues.org/index316.html, consulté le 15 juin 2011.

77. Sa thèse, Music at Cluny. The tradition of Gregorian chant for the proper of the mass, melodic variants and microtonal nuances, a été soutenue à Princeton un peu avant notre période de référence, en 1998.

78. Par exemple : Frederick S. Paxton, « Performing Death and Dying at Cluny in the High Middle Ages ", dans Practicing Catholic. Ritual, Body and Contestation in Catholic Faith, Bruce T. Morrill, Joanna E. Ziegler et Susan Rodgers éds., New York, 2006, p. 43-52 et surtout "Death by Customary at Eleventh-Century Cluny ", dans From Dead of Night to End to Day, op. cit., p. 297-318.

79. I. Cochelin, "Étude sur les hiérarchies monastiques : le prestige de l'ancienneté et son éclipse à Cluny au xi ${ }^{\mathrm{e}}$ siècle ", Revue Mabillon, t. 72, 2000, p. 5-37, EAD. et S. Boynton, "The Sociomusical Role of Child Oblates at the Abbey of Cluny in the Eleventh Century ", dans Musical Childhoods and the Cultures of Youth, S. Boynton et Roe-Min KoK éds., Middleton (Connecticut), 2006, p. 3-24.

80. Cf. Scott G. Bruce, Silence and Sign Language in Medieval Monasticism. The Cluniac Tradition c. 900-1200, Cambridge/New York, 2007.

81. Jörg SonNTag, Klosterleben im Spiegel des Zeichenhaften. Symbolisches Denken und Handeln hochmittelalterlicher Mönche zwischen Dauer und Wandel, Regel und Gewohnheit, Berlin, 2008 (Vita regularis. Abhandlungen, 35). 
ou celui de Mirko Breitenstein sur le noviciat ${ }^{82}$. De la sorte, Cluny demeure un point d'ancrage historiographique important pour des enquêtes plus vastes - bien entendu à la mesure de ce qui a été son importance et son influence sociale. Parmi les secteurs prometteurs à cet égard, il faut mentionner celui des textes de nature normative, qu'ils aient trait à la règle, aux coutumes ou aux statuts clunisiens - et, là encore, le renouveau vient des travaux sur Cluny mais pas exclusivement, si l'on en juge par les enquêtes conduites en Allemagne sur la pragmatische Schriftlichkeit d'une part, et sur l'institution comme objet historique et sociologique d'autre part, dont relèvent les deux ouvrages qui viennent d'être cités. Ces recherches sur l'écrit pratique ou " pragmatique » et sur les voies du contrôle institutionnel invitent en effet à se pencher sur les textes qui organisent, réglementent et hiérarchisent, et c'est dans ce sillage que trouvent leur place logique les ouvrages publiés notamment par Cristina Andenna et G. Melville ${ }^{83}$ ou encore par I. Cochelin et S. Boynton. Bien entendu, certains sujets dont l'exploration avait commencé il y a un certain temps continuent de faire l'objet de travaux et de publications, que ce soit sur les coutumiers ${ }^{84}$, ou, dans une moindre mesure, sur la memoria et la commémoration des défunts, qui demeure un lieu de découvertes et d'intéressantes collaborations, comme celle qui a déjà été signalée entre Fr. Neiske et C. M. Reglero de la Fuente ${ }^{85}$.

Au moment de dresser un bilan final de ce panorama, on est tenté de se demander ce qui, en fin de compte, caractérise l'historiographie clunisienne par rapport à celles d'autres ordres religieux ou à d'autres domaines de la recherche. Il a en effet souvent été question, dans les lignes qui précèdent, de questionnements s'inscrivant dans des mouvements ou des problématiques larges. En fait, l'impression se dégage que ce qui est, ou pourrait être, spécifiquement clunisien, c'est le rôle de creuset joué par le mouvement d'écriture de l'histoire des moines noirs et de leur environnement. La situation historique et historiographique très particulière de Cluny a pour corollaire le fourmillement des questionnements méthodologiques et épistémologiques dans les études qui lui sont consacrées. Ceci se révèle de deux manières: de manière synchronique, tant les réflexions de méthode constituent l'accompagnement habituel des travaux sur Cluny; de manière pour ainsi dire diachronique, dans la mesure où bien des chercheurs ont pu prendre appui sur leur expérience clunisienne pour la dépasser et l'élargir. Si l'on suit les parcours des chercheurs évoqués dans cette revue des travaux récents, dans ceux de D. Iogna-Prat et de G. Melville déjà mentionnés, et aussi dans ceux de D. Méhu ${ }^{86}$, J. Oberste ${ }^{87}$,

82. Mirko Brettenstern, Das Noviziat im hohen Mittelalter. Zur Organisation des Eintrittes bei den Cluniazensern, Cisterziensern und Franziskanern, Münster, 2009 (Vita regularis. Abhandlungen, 38).

83. Regulae, Consuetudines, Statuta. Studi sulle fonti normative degli ordini religiosi nei secoli centrali del Medioevo, Cr. Andenna et G. Melville éds., Münster, 2005 (Vita regularis. Abhandlungen, 25); je me permets d'y renvoyer à S. BARRET, "Regula Benedicti, consuetudines, statuta : aspects du corpus clunisien ", aux p. 65-104.

84. Le recueil From Dead of Night to End of Day, op. cit., doit justement ètre poursuivi par un deuxième volume sous la direction de Carolyn Malone et Clark Maines; I. Cochelin a récemment publié "Le pour qui et le pourquoi (des manuscrits) des coutumiers clunisiens ", dans Ad libros! Mélanges d'études médiévales offerts à Denise Angers et Claude Poulin, Jean-François Cottier, Sébastien Rossignol et Martin Gravel éds., Montréal, 2010, p. 121-38.

85. Cf. supra, n. 38.

86. Après avoir envisagé Cluny sous l'angle de larges problématiques, il a diversifié et élargi son objet d'étude, comme l'atteste par exemple le volume collectif Mises en scène et mémoires de la consécration de l'église dans l'Occident médiéval, D. MéHu dir., Turnhout, 2008 (Collection d'études médiévales de Nice, 7).

87. Ses travaux portent notamment sur l'histoire de l'hérésie et sur l'histoire urbaine : ainsi, J. Oberste, Der Kreuzzug gegen die Albigenser : Ketzerei und Machtpolitik im Mittelalter, Darmstadt, 2003, et son entreprise de publication Die mittelalterlichen Stadtbücher Dresdens (1404-1535) und Altendresdens (1412-1528). Kritische Edition und Kommentar, J. OвERste et Thomas KüBler éds., Leipzig, 2007-2010, 4 vol. prévus dont les t. I, II et IV sont parus. 
Fl. Cygler ${ }^{88}$ et E. Magnani ${ }^{89}$, Cluny trouve sa place comme une phase, importante mais non pas unique, de la construction de leur démarche scientifique. Il ne s'agit pas, en le constatant, de faire d'une spécialisation continue une quelconque marque d'infériorité. En revanche, ces similitudes d'expérience suggèrent les potentialités offertes par les recherches sur les moines noirs, non pas tant du fait de l'objet lui-mème que gràce à tout ce qui a pu et peut être construit autour de lui. Ne peut-on pas, d'ailleurs, y trouver aussi l'explication d'impressions ressenties, et sans doute fausses, d'un certain ralentissement des recherches nouvelles au profit de la constitution de bilans ? Sans doute, les centres de gravité qui s'étaient imposés il y a quelques années sont aujourd'hui moins perceptibles - du moins, d'un point de vue clunisiocentré. Sans doute, le nombre des publications a tendance à se réduire - mais en réalité, assez peu, et sans doute moins que le phénomène de dispersion des travaux ne le laisse croire. Inversement, le gain scientifique apporté par les études clunisiennes a fécondé et féconde bien d'autres recherches et bien d'autres secteurs, au gré de la chronologie des cheminements persomnels. C'est aussi, du reste, cette chronologie forcément différenciée qui détermine en partie le flux et le reflux des publications. En d'autres termes, pour apprécier au mieux l'état actuel des études clunisiennes, il est peut-ètre également bon de dépasser leurs limites, et de constater qu'à l'intérieur du cercle aussi bien qu'à l'extérieur, elles continuent à porter de beaux et bons fruits, que ce soient les travaux en cours, ou les colloques et les rencontres dont ce volume de la Revue Mabillon propose aussi le bilan. Le constater ne vaut-il pas mieux que de se trouver en présence d'une clunisiologie qui se ferait par trop exclusive et, certainement, perdrait de sa créativité?

Sébastien BARRET 